\title{
Análise de casos cirúrgicos para o tratamento de hemorragia subaracnóidea aneurismática
}

\section{Analysis of Surgical Cases for the Treatment of Subarachnoid Hemorrhage Aneurysmal}

\author{
Sheila Cristiane Evangelista Creôncio ${ }^{1}$ José Carlos de Moura ${ }^{2}$ Bruno Lázaro Ramos Rangel ${ }^{3}$ \\ Márcio Felipe Bastos Coelho ${ }^{4}$ Thadeu Borges Souza Santos ${ }^{5}$ Maria Andréia Lopes de Freitas ${ }^{6}$ \\ ${ }^{1}$ Enfermeira especialista em urgência. Mestranda em ciências da \\ saúde e biológicas pela Universidade Federal do Vale do São \\ Francisco (Univasf), Petrolina, PE, Brasil \\ 2 Professor Doutor da Univasf, Petrolina, PE, Brasil \\ ${ }^{3}$ Graduado em Medicina pela Univasf, Petrolina, PE, Brasil \\ ${ }^{4}$ Enfermeiro residente em enfermagem-urgência na UnivasfNIVASF, \\ Petrolina, PE, Brasil \\ ${ }^{5}$ Enfermeiro intensivista e Auditor, Professor Assistente do \\ Departamento de Ciências da Vida - Universidade do Estado da \\ Bahia, Salvador, BA, Brasil \\ ${ }^{6}$ Enfermeira, Doutora pela Universidade Federal de São Paulo \\ (Unifesp), São Paulo, SP, Brasil. Pós-doutoranda na Universidade de \\ Leipzig, Alemanha \\ Address for correspondence José Carlos de Moura, PhD, Rua Joaquim \\ Nabuco, 798, Centro, Petrolina, PE, Brasil, CEP 56304-040 \\ (e-mail: jcdemoura@uol.com.br). \\ Serviço de Neurocirurgia do Hospital de Ensino da Universidade \\ Federal do Vale do São Francisco (Univasf), Petrolina, PE, Brasil
}

\section{Resumo}

\section{Palavras-Chave}

- hemorragia subaracnóidea

- aneurisma

- neurocirurgia
Objetivo Avaliar o perfil epidemiológico, os resultados cirúrgicos e os casos de hemorragia subaracnóidea aneurismática ocorridos no Hospital de Ensino da Univasf. Métodos Estudo descritivo e analítico, com abordagem quantitativa, baseado no caráter não experimental, documental e retrospectivo, os prontuários dos pacientes com diagnóstico de hemorragia subaracnóidea aneurismática não traumática admitidos em um hospital de ensino de setembro de 2008 a setembro de 2012.

Resultados Verificou-se uma prevalência de hemorragia subaracnóidea aneurismática em Petrolina de 1,8 para cada 10 mil habitantes. Dos 55 prontuários, o sexo feminino foi o mais acometido (61,81\%), com faixa etária de 41 a 50 anos. Aneurismas múltiplos foram encontrados em $23,5 \%$. Com relação às escalas de Hunt-Hess e Fisher, obtivemos respectivamente $40 \%$ com Hunt-Hess grau dois e $35,7 \%$ com Fisher grau três. Não apresentaram nenhum tipo de déficit neurológico desde a admissão até a alta hospitalar $54,8 \%$ dos pacientes, e somente $1,81 \%$ apresentaram déficit neurológico após o procedimento cirúrgico. A reabordagem cirúrgica foi necessária em 3,7\% dos casos. A mortalidade cirúrgica foi de $3,5 \%$.

Conclusão Existe uma prevalência de hemorragia subaracnóidea aneurismática em Petrolina/PE de 1,8 por 10 mil habitantes. received

April 1, 2014

accepted

November 1, 2014
DOI http://dx.doi.org/

$10.1055 / \mathrm{s}-0035-1547374$ ISSN $0103-5355$.
Copyright @ 2015 Thieme Publicações

Ltda, Rio de Janeiro, Brazil
License terms

c) $(1) \$$ 
Abstract

\section{Keywords \\ - subarachnoid hemorrhage \\ - aneurysm \\ - neurosurgery}

Objective To evaluate the epidemiological profile, the surgical results and cases of Subarachnoid Hemorrhage Aneurysmal occurred in the UNIVASF Teaching Hospital. Methods A descriptive and analytical study with a quantitative approach based on non-experimental, documentary and retrospective study, the charts of patients with nontraumatic Subarachnoid Hemorrhage Aneurysmal admitted to a teaching hospital from September 2008 to September 2012.

Results It has been found a prevalence of Subarachnoid Hemorrhage Aneurysmal in Petrolina 1.8 per 10 thousand inhabitants. Of the 55 records, females were more prevalent $61.81 \%$, ranging in age from 41 to 50 years; $23.5 \%$ had multiple aneurysms. Regarding Hunt-Hess and Fisher scales, respectively obtained $40 \%$ with Hunt-Hess grade two and $35.7 \%$ with Fisher grade three. $54.8 \%$ did not experience any neurological deficit from admission to discharge, and only $1.81 \%$ had neurological deficits after surgery; $3.7 \%$ of cases needed to be surgically re-approached. Operative mortality was $3.5 \%$.

Conclusion There is a prevalence of Subarachnoid Hemorrhage Aneurysmal in Petrolina/PE 1.8 per 10,000 inhabitants.

\section{Introdução}

No ano de 2004, as doenças cerebrovasculares foram responsáveis por $9,7 \%$ dos óbitos, sendo a segunda causa de mortalidade no mundo. De acordo com estimativas recentes, existe uma tendência ao aumento progressivo do número de óbitos por doenças cerebrovasculares, e a estimativa é que em 2030 o percentual chegue a $12,1 \%$ da mortalidade mundial. ${ }^{1,2}$

A hemorragia subaracnóidea aneurismática (HSA) é uma alteração neurológica que muitas vezes representa uma emergência neurocirúrgica ocasionada por um sangramento abrupto no espaço subaracnóideo, onde geralmente o sangue fica limitado ao espaço do líquido cefalorraquidiano (LRC), compreendido entre as membranas pia-máter e aracnoide. A ruptura de aneurismas cerebrais é a causa mais comum de hemorragia não traumática subaracnóidea. A mortalidade é alta neste grupo de pacientes, e metade deles falecem antes mesmo de terem acesso a um atendimento hospitalar. ${ }^{1-3}$

Nos indivíduos com HSA, a taxa de mortalidade chega a alcançar índices de $40 \%$, sendo que esta é responsável por $5 \%$ de todos os eventos de disfunção neurológica em que a manifestação inicial acontece de forma súbita. 1,3,4

Com o aumento da idade, as chances de um aneurisma romper aumentam, sendo que o pico de incidência ocorre por volta dos 60 anos de idade. ${ }^{2}$

Na forma espontânea, os aneurismas correspondem a $75-80 \%$ das hemorragias, e as malformações arteriovenosas representam 4-5\% desses eventos. Existem outras situações menos frequentes como vasculites que envolvem o sistema nervoso central (SNC), distúrbios de coagulação, tumores, dissecção de artéria cerebral e doença falciforme, entre outras, inclusive por causas desconhecidas em $14-22 \%$ dos casos. $^{3,5}$
A ruptura de um aneurisma encefálico apresenta alta mortalidade no primeiro episódio, e aproximadamente $50 \%$ evoluem para óbito antes do primeiro atendimento. Os sobreviventes apresentam um risco de ressangramento de $60 \%$ nos 3 meses seguintes. ${ }^{6}$ A abordagem microcirúrgica é confirmada como uma boa opção de tratamento. Após alguns estudos, houve uma tendência mundial para o tratamento endovascular, e este tratamento favoreceu o condução terapêutica nos aneurismas não rotos. ${ }^{7}$

$\mathrm{O}$ fato de ainda não existirem dados epidemiológicos acerca da HSA não traumática na região do Vale do São Francisco nos levou a optar pela avaliação do perfil e do desfecho dos casos de hemorragia subaracnóidea aneurismática (HSA) ocorridos no Hospital de Ensino da Universidade Federal do Vale do São Francisco (HE-Univasf).

\section{Métodos}

Foram estudados os prontuários dos pacientes admitidos no serviço do HE-Univasf acometidos por Hemorragia Subaracnóidea Aneurismática, na região do Vale do São Francisco, entre setembro de 2008 e setembro de 2012. 0 protocolo de pesquisa foi aprovado pelo Comitê Institucional de Ética em Pesquisa da Univasf ( ${ }^{\circ}{ }^{00008 / 190313}$ Cedep/Univasf).

As seguintes variáveis foram analisadas conforme o protocolo estabelecido: sexo, idade, localização do aneurisma, número de aneurismas por paciente, classificação Hunt-Hess e Fisher, déficit neurológico antes e depois do procedimento neurocirúrgico, exames de imagem realizados, reabordagem cirúrgica, evolução/desfecho, e óbito anterior ou posterior à cirurgia.

Após a coleta das informações, elas foram submetidas a uma análise estatística descritiva, em que os dados foram transferidos para planilhas do Microsoft Excel ${ }^{\circledR}$ e Microsoft 


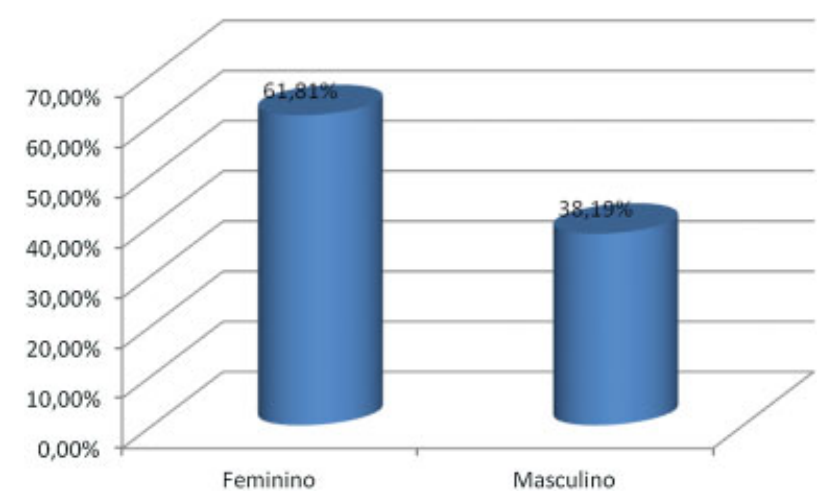

Fig. 1 Apresentação quantitativa sobre o sexo dos pacientes com diagnóstico de aneurisma cerebral.

Word ${ }^{\circledR}$, para construção dos gráficos e tabelas, contendo números absolutos e percentuais seguidos de análise e discussão dos resultados.

Durante a realização desta pesquisa, não foram declarados conflitos de interesse associados à realização deste estudo.

\section{Resultados}

Esta pesquisa contou com 55 prontuários de pacientes diagnosticados com hemorragia subaracnóidea aneurismática. A idade dos pacientes variou entre 20 e 90 anos. 0 sexo

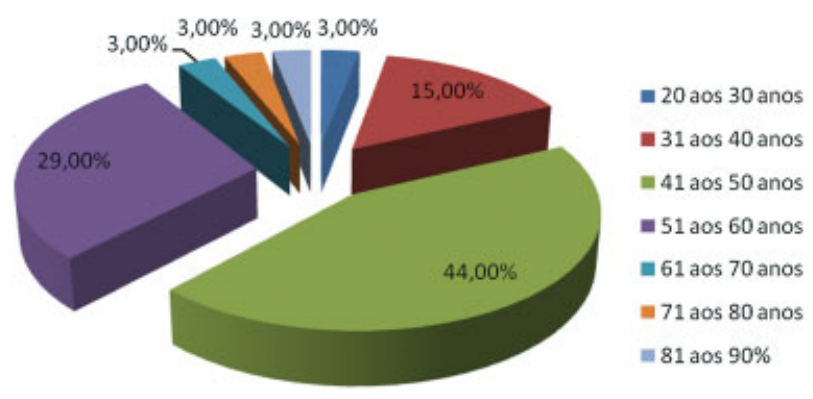

Fig. 2 Apresentação quantitativa sobre a idade dos pacientes com diagnóstico de hemorragia subaracnóidea aneurismática.

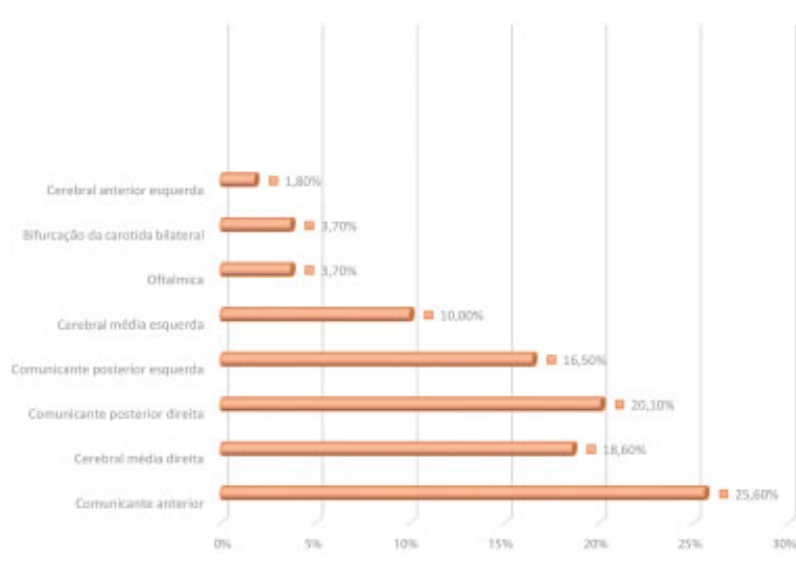

Fig. 3 Apresentação quantitativa sobre a localização dos aneurismas nos pacientes avaliados.

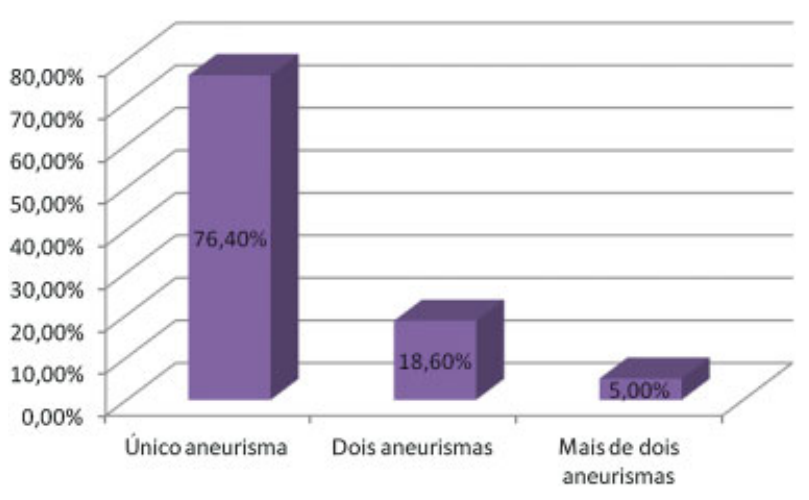

Fig. 4 Apresentação quantitativa sobre o número de aneurismas em cada paciente.

feminino foi o mais acometido $(61,81 \%)$ ( - Fig. 1), e a faixa etária de 41 a 50 anos foi a mais acometida (44\%) (-Fig. 2).

A maioria dos aneurismas foi encontrada na artéria cerebral média direita (18,6\%), seguida respectivamente pelas artérias: comunicante posterior direita (20,1\%), comunicante posterior esquerda (16,5\%), comunicante anterior (25,6\%), cerebral média esquerda (10\%), cerebral média direita (18,6\%), oftálmica, bifurcação da carótida bilateral (cada uma com $3,7 \%$ ) e cerebral anterior esquerda (apenas 1,8\%) (-Fig. 3). Do total de casos, 23,5\% apresentavam aneurismas múltiplos, sendo que $18,6 \%$ apresentavam dois aneurismas e $5 \%$ apresentavam mais de dois aneurismas (-Fig. 4).

Neste estudo, $40 \%$ dos pacientes foram classificados com Hunt-Hess grau dois, seguidos por Hunt-Hess grau um $(30,2 \%)$, Hunt-Hess grau três $(22,7 \%)$ e Hunt-Hess grau quatro (7,1\%) (-Fig. 5).

Em nossa amostra, 35,7\% dos casos foram classificados como Fisher grau três (o que significa dizer que estes possuíam coágulo subaracnóideo localizado espesso > $1 \mathrm{~mm}$ ), $29 \%$ como Fisher grau dois, 26\% como Fisher grau um e 9,3\% como Fisher grau quatro, conforme ilustrado na - Fig. 6 .

Os prontuários analisados mostraram que 54,8\% dos pacientes não apresentaram nenhum tipo de déficit neurológico desde a admissão até a alta hospitalar. Entre os que apresentaram déficit neurológico, apenas $1,81 \%$ foram acometidos após o procedimento cirúrgico ( - Fig - 7).

Todos os pacientes foram submetidos ao exame de angiografia cerebral, $72,34 \%$ deles ao exame de tomografia

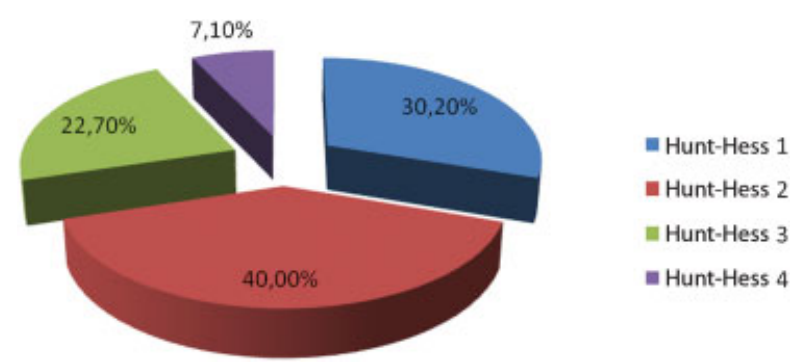

Fig. 5 Apresentação quantitativa sobre a escala de Hunt-Hess nos pacientes avaliados. 


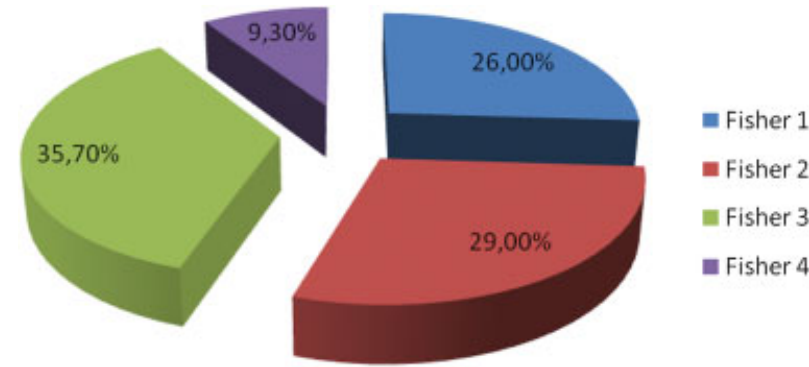

Fig. 6 Apresentação quantitativa sobre a escala de Fisher nos pacientes avaliados.

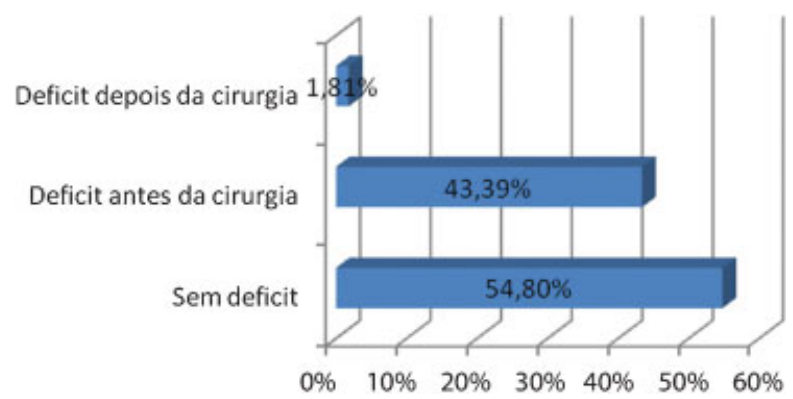

Fig. 7 Apresentação quantitativa sobre a presença de déficit antes e depois da cirurgia.

computadorizada de crânio, e somente $14,89 \%$ ao exame de ressonância magnética ( - Fig . 8).

Apenas 3,7\% dos casos precisaram de reabordagem cirúrgica (-Fig . 9).

O tempo médio entre a admissão e a realização do procedimento encontrado nesta casuística foi de 20,9 dias, sendo que $94,73 \%$ dos pacientes receberam alta hospitalar, $1,7 \%$ foram transferidos para outros serviços de saúde e 3,5\% evoluíram para óbito ${ }^{8}$ - Fig . 10).

\section{Discussão}

Em um estudo de pacientes com hemorragia subaracnóidea aneurismática espontânea realizado por Loureiro, ${ }^{5}$ prevaleceu a faixa etária de 35 a 74 anos, com média de 51,62 anos. Em nosso estudo, houve prevalência na faixa etária de 41 a 50 anos (44\%).

Em nossa amostra, à semelhança de outros estudos publicados, ${ }^{3,5,6,9-11}$ houve prevalência de aneurisma cerebral entre as mulheres $(61,81 \%)$.

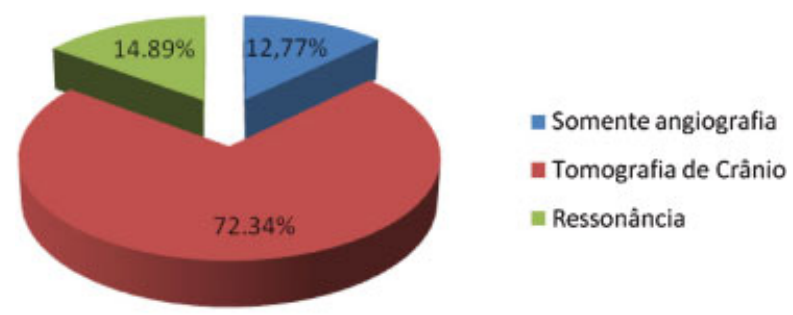

Fig. 8 Apresentação quantitativa sobre os exames de imagem realizados nos pacientes com aneurisma; todos realizaram angiografia.

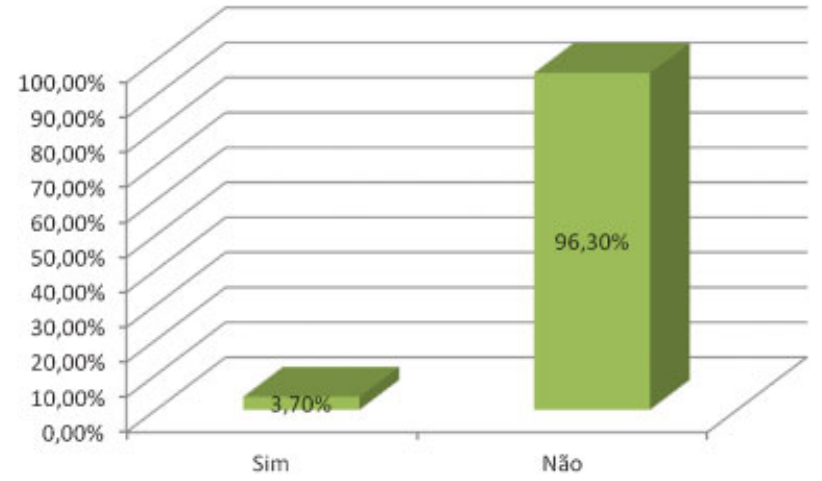

Fig. 9 Apresentação quantitativa sobre reabordagem cirúrgica.

Como em outros estudos, em nossa amostra houve prevalência dos aneurismas na artéria cerebral média. ${ }^{3,5}$ Outros estudos mostram que de 20 a $30 \%$ dos pacientes apresentaram aneurismas múltiplos, e nossa pesquisa corroborou este resultado, encontrando $23,5 \%$ de pacientes com aneurismas múltiplos (18,6\% com dois aneurismas e $5 \%$ com mais de dois aneurismas). ${ }^{3,5}$

A escala de Hunt-Hess é o sistema clínico de classificação da HSA mais comumente utilizado, por meio da qual se avalia a condição clínica do paciente. ${ }^{5}$

Neste estudo, $40 \%$ dos pacientes foram classificados com Hunt-Hess grau dois, seguidos por Hunt-Hess grau um com $30,2 \%$, Hunt-Hess grau três com $22,7 \%$ e Hunt-Hess grau quatro com 7,1\%. Estudo realizado por Loureiro ${ }^{5}$ também apresentou um número maior de Hunt-Hess grau dois, com $53,85 \%$ dos casos.

A escala de Fisher baseia-se na quantidade de sangue acumulado nas cisternas da base, ventrículos e parênquima cerebral, vistos na tomografia computadorizada, para predizer o risco do desenvolvimento de vasoespasmo clínico e angiográfico. ${ }^{2}$ Desta amostra, $35,7 \%$ dos casos foram classificados como Fisher grau três, o que significa dizer que estes possuíam coágulo subaracnóideo localizado espesso $>1 \mathrm{~mm}$, seguidos por $29 \%$ dos casos com Fisher grau dois, 26\% com Fisher grau um e 9,3\% com Fisher grau quatro.

Apenas 3,7\% dos casos precisaram de reabordagem cirúrgica. $\mathrm{O}$ ressangramento está associado a uma alta taxa de morbidade e mortalidade. ${ }^{3,12}$ Deve-se suspeitar de ressangramento ou vasoespasmo em todo paciente que não foi tratado neurocirurgicamente e que apresente piora

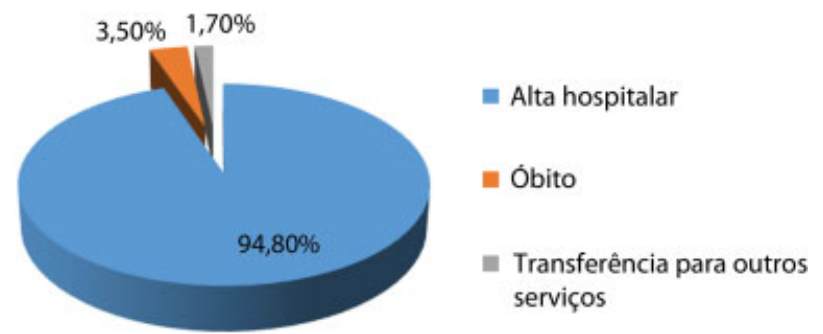

Fig. 10 Apresentação quantitativa sobre o desfecho dos casos de pacientes com aneurisma que foram submetidos à neurocirurgia. 
neurológica súbita. Dos pacientes com HSA aneurismática, $30 \%$ sangram novamente durante o primeiro mês, sendo que esta incidência é mais alta nas duas primeiras semanas após o sangramento inicial. ${ }^{3}$

O tempo médio entre a admissão e a realização do procedimento encontrado nesta casuística foi de 20,9 dias. Este fato deve-se à atual impossibilidade operacional para as cirurgias precoces; além disto, estudo recente demonstra em seus resultados que a cirurgia tardia vem apresentando melhores resultados que a cirurgia precoce. ${ }^{8,13}$ Neste período, tivemos quatro óbitos por ressangranmentos no préoperatório.

De acordo com um estudo realizado na Alemanha, sabe-se que a morbimortalidade é de $10 \%$, independente da forma de terapia e apesar da evolução tecnológica. ${ }^{13}$

Em um estudo realizado em Curitiba com uma amostra de 93 pacientes operados, verificou-se que $73 \%$ pertenciam ao sexo feminino e que a mortalidade (somados os casos de pré e pós-operatória) foi de $32 \%{ }^{13}$ Um grupo de Porto Alegre publicou resultados sobre uma amostra de 88 pacientes operados em que a mortalidade global foi de 7,9\%. ${ }^{14}$

Em um estudo realizado por Borges e Gallani, ${ }^{15}$ pacientes submetidos ao tratamento cirúrgico obtiveram $76 \%$ de bons resultados pós-cirúrgicos e uma taxa de mortalidade de $8 \%$ (4 casos em 38 pacientes). Em nosso estudo estudo, 88,7\% dos pacientes receberam alta hospitalar, 1,8\% dos pacientes foram transferidos para outros serviços de saúde e 3,5\% evoluíram para óbito. Segundo Turcato et al., ${ }^{3}$ em indivíduos com HSA com mais de 60 anos a mortalidade foi de $48 \%$, enquanto em indivíduos jovens esta taxa foi de $19 \%$.

Quase todos os pacientes desta pesquisa foram abordados por microcirurgia. Dois pacientes com aneurisma de oftálmica e os pacientes com aneurisma da circulação posterior foram encaminhados para embolização. Em recente estudo realizado pelo grupo de Spetzler et al., ${ }^{7}$ verificou-se que a opção pela microcirurgia apresenta melhor oclusão do aneurisma e menor incidência de recidiva após 3 anos de acompanhamento, embora a curto prazo (1 ano) os resultados se apresentem invertidos. Entretanto, a microcirurgia permanece como primeira opção para: pacientes jovens, a maioria dos aneurismas da cerebral média e os de colos largos. No entanto, existe atualmente, em muitos centros de neurocirurgia do mundo, uma progressiva tendência para se oferecer o procedimento endovascular como primeira escolha para o tratamento. $^{7}$

\section{Conclusão}

Este estudo revelou uma prevalência de pacientes acometidos com HSA de 1,8 a cada 10 mil habitantes no município de Petrolina/PE. O resultado cirúrgico no Hospital de Ensino da Univasf em Petrolina apresentou 95,25\% de bons resultados.

\section{Conflitos de Interesse}

Os autores declaram não haver conflitos de interesse.

\section{Agradecimentos}

Os autores agradecem aos funcionários do Hospital de Ensino da Universidade Federal do Vale do São Francisco (HE-Univasf) pela colaboração na realização desta pesquisa.

\section{Referências}

1 Mansur AP, de Souza MF, Timerman A, Avakian SD, Aldrighi JM, Ramires JA. Trends in the risk of death from cardiovascular, cerebrovascular and ischemic diseases in thirteen States of Brazil from 1980 to 1998. Arq Bras Cardiol 2006;87(5):641-648

2 Pontes-Neto OM, Oliveira-Filho J, Valiente R, et al. Brazilian guidelines for the management of intracerebral hemorrhage. Arq Neuropsiquiatr 2009;67(3B):940-950

3 Turcato C, Pereira SW, Ghizoni MF. Hemorragia Subaracnóide. ACM Arq Catarin Med 2006;35(2):78-84

4 Mangieri P, Suzuki K, Ferreira M, Domingues L, Casulari LA. Evaluation of pituitary and thyroid hormones in patients with subarachnoid hemorrhage due to ruptured intracranial aneurysm. Arq Neuropsiquiatr 2003;61(1):14-19

5 Loureiro AB. Análise dos resultados da reabilitação em pacientes com hemorragia subaracnóide aneurismática. [dissertação]. Campinas: Universidade Estadual de Campinas; 2007

6 Machado FS, Akamine N. Hemorragia subaracnóidea. In: Knobel E, et al. Terapia intensiva: neurologia. São Paulo: Atheneu; 2002: 123-136

7 Spetzler RF, McDougall CG, Albuquerque FC, et al. The Barrow Ruptured Aneurysm Trial: 3-year results. J Neurosurg 2013; 119(1):146-157

8 Ji Y, Meng QH, Xu Z, Zhang QL, Wang ZG. [Correlation of surgical timing and prognosis for ruptured intracranial aneurysms]. Zhonghua Yi Xue Za Zhi 2012;92(13):924-926

9 Ostbye T, Levy AR, Mayo NE. Hospitalization and case-fatality rates for subarachnoid hemorrhage in Canada from 1982 through 1991. The Canadian Collaborative Study Group of Stroke Hospitalizations. Stroke 1997;28(4):793-798

10 Aristizabal JHM, Diez JCP. Hemorragia subaracnoidea espontânea. ECM 2002;7(2):32-47

11 Borges G. Tratamento cirúrgico e fatores preditivos da hemorragia subaracnóide [tese]. Campinas: Universidade Estadual de Campinas; 2003

12 Mayer PL, Awad IA, Todor R, et al. Misdiagnosis of symptomatic cerebral aneurysm. Prevalence and correlation with outcome at four institutions. Stroke 1996;27(9):1558-1563

13 Steiger HJ. A view on the current and future therapy of brain aneurysms. Acta Neurochir Suppl 2011;112:71-75

14 Zamponi JO Junior, Silva PE, Ramos GZ, Buchaim GM, Andrade LC, Sala LF. Analysis of intracranial aneurysms treated at a University Hospital in Curitiba. Arq Bras Neurocir 2012;31(2): 55-60

15 Borges G, Gallani NR. Cerebral aneurysms. Assessment of 50 cases operated on and comparison with previous series. Arq Neuropsiquiatr 1997;55(2):287-291 